\title{
AGENT-BASED INFRASTRUCTURE OF SUPPLY CHAIN NETWORK MANAGEMENT
}

Charu Chandra ${ }^{1}$, Alexander V.Smirnov ${ }^{1,2}$, Leonid B. Sheremetov ${ }^{2,3}$

${ }^{\prime}$ Industrial and Manufacturing Systems Engineering Department University of Michigan - Dearborn 4901 Evergreen Road, Dearborn, Michigan, 48128-1491, U.S.A. e-mail: charu@umich.edu

${ }^{2}$ St. Petersburg Institute for Informatics and Automation of the Russian Academy of Sciences 39, $14^{\text {th }}$ Line, St. Petersburg, 199178, RUSSIA e-mail:smir@mail.iias.spb.su

${ }^{3}$ Computer Science Research Center, National Technical University (CIC-IPN), Av. Juan de Dios Batiz esq. Othon de Mendizabal s/n Col. Nueva Industrial Vallejo, México, D.F., C.P. 07738, MEXICO e-mail: sher@cic.ipn.mx

\begin{abstract}
Supply-chain network (SCN), a society formed by autonomous agents to solve a common logistics problem is a philosophy for enterprise integration. A multi agent infrastructure for information support of SCN management is proposed. It consists of SCN problem domain agents and an agent platform. Member acts as a Problem domain agent, while Group serves as a Supply Chain Advisor (SCA). An agent platform consists of Directory Facilitator, Agent Management System, Agent Communication Channel, Internal Platform Message Transport, and Wrapper Agents. Use of this platform ensures interoperability among agents and reusability of components and services.
\end{abstract}

\section{INTRODUCTION}

In order to understand the significance of changes taking place in enterprise integration initiatives, it would be prudent to review trends in production and operations management activities (Chandra and Kumar, 2000; IMTR, 1999; ISO TC 184/SC 5/WG 1, 1997; NIST 1999; and Sousa et al., 1999). Supply-chain network ( $\mathrm{SCN}$ ) is a philosophy for enterprise integration. It is a society (network of members, termed a group) formed by autonomous agents to solve a common logistics problem. Management processes and their utility within a supply chain network can be described in the context of a product life cycle viz., forecast management, inventory management, sourcing, production, warehousing, and transportation. 
Figure 1 offers template of a generic supply chain. It has a demand and a supply echelon. Its structure is contingent upon how these echelons are configured, separately as well as jointly.

Figure 2 depicts a textile supply chain that shows the integration of these echelons. The problem of synchronizing flows associated with these echelons maximally, is the configuration management problem for the supply chain. It can be formulated in the following way: to dynamically configure a supply chain from a number of available "members" (each one described by the services provided) that generate plans for the supply chain production to meet consumer demand (marketing), while making effective use of resources and promoting cooperation among members, so as to achieve reduction in lead time and inventory costs. Integrated Production Planning and Control is a combination of philosophies, concepts, and tools and techniques, to manage these deviations in expectations from the demand and supply functions of a productive system. The challenge is configuring the supply chain flexibly, while maintaining optimal information flow.

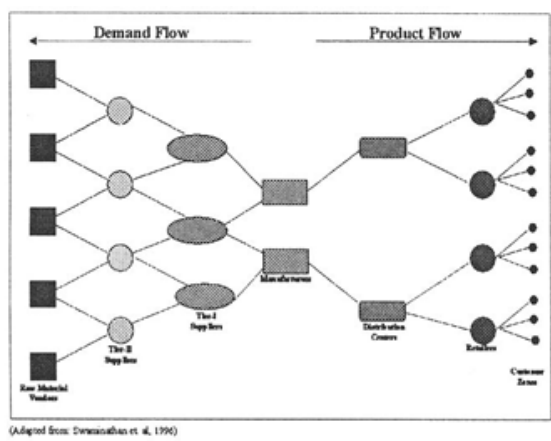

Figure 1. A Generic Supply Chain template

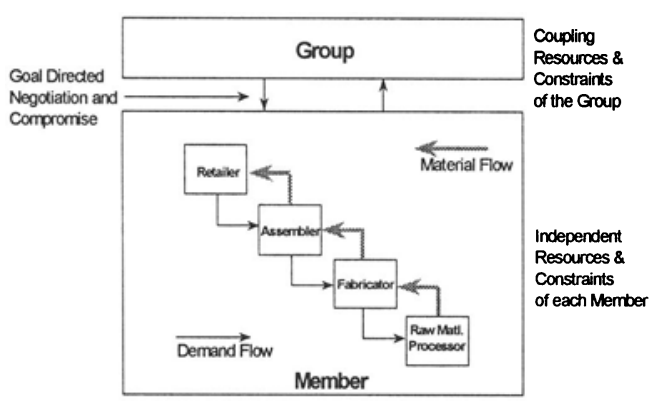

Figure 2. A Textile Supply Chain representation

Multi agent systems (MAS), where agents cooperate to achieve common objective(s), is one of the most promising implementation platforms for information support of SCN management, where each member may have conflicting objectives but also can modify its norms of behavior to accommodate other member's perspectives (Banerjee and Smirnov, 1999; Chandra and Kumar, 2000; Chen, 1999; Groumpos and Smirnov, 1998; and Moulin and Chaib-Draa, 1996). With their collaborative efforts, agents sustain the progress of each member as well as the group. The purpose of the MAS proposed in this paper is to support design of different topologies for the SCN, while minimizing time and costs and avoiding (operational) bottlenecks. Conflicting objectives of SCN members is the reason for a satisficing or Pareto-optimal solution(s).

The rest of the paper is organized as follows. Main principles of information support technologies for SCN management is introduced in section 2 and the general framework is described in section 3. The deployment of the overall MAS architecture is described in section 4. An interaction scenario is discussed in section 5. Finally conclusions and extensions of this research are discussed in section 6. 


\section{INFORMATION SUPPORT TECHNOLOGIES FOR SCN}

The proposed SCN management technologies are categorized into two groups, (i) problem solving, and (ii) information support. For the first group, these are (1) custom-ordered (mass customization) management, (2) configuration management, and (3) constraint satisfaction and propagation. For the second group, these are (1) data and knowledge management, (2) multi-agent and intelligent agent, and (3) conceptual and information modeling. Information support technologies for configuration management is described below.

Data and Knowledge Management: Intelligent supports for the SCN management approach is critical in realizing competitive advantage for networking organizations. Knowledge is key to managing collaborative activities within and between SCNs. Therefore, knowledge must be relevant to overall business goals and processes and be accessible in the right form and at the right time. This is accomplished via design and development of Knowledge Management at following knowledge levels:

- System knowledge describes rules for integration between SCN units and its management and maintenance.

- Facilitator knowledge describes rules for distribution of knowledge and identification of access level in sharing data and knowledge base.

- Unit knowledge describes reusable methods, techniques and solutions for problem solving at the unit level.

- User knowledge describes knowledge related to individualized special skills of a user at the problem domain level.

The methodology suggested in this paper is limited to designing SCN configurations for product-process-resource (PPR) systems and focused on utilizing reusable knowledge through ontological descriptions. It is based on GERAM, the Generalized Enterprise Reference Architecture and Methodology (ISO TC 184/SC 5/WG 1, 97). Applying the GERAM methodology enables forming the conceptual model of the SCN. This is accomplished by knowledge modeling its product, process, and resource components to satisfy manufacturing constraints in its environment. The implementation of e-management approach is based on the shared information environment that supports the PPR model used for integration and coordination of user's (unit's) activity. This model is studied from various viewpoints of user (unit) groups. Reusable knowledge management is a concept of knowledge management to organize "knowledge clusters" by their inherently common characteristics observed in various problem domains. These clusters are utilized as templates to describe unique conceptual models of an enterprise, or its components.

Ontology is a form of knowledge representation applied in various domains. It is useful in creating unique models of a SCN by developing specialized knowledge bases specific to various e-management problem domains. Ontologies are managed by translation and mapping between different types of entities and attributes. 
Ontological translation of an enterprise, such as a supply chain is necessary because networks are multi-ontology classes of entities. Various ontologies for an entity describe its unique characteristics in context with the relationship acquired for a specific purpose or problem.

Ontology design is based on an ontology hierarchy. The top-level ontology is the "shared ontology" for domain independent representation of the problem set. This type of ontology is needed to describe an abstract model using common knowledge model representation. The lower-level ontology is an "application ontology", and is a combination of the "domain specific ontology" and the "problemspecific ontology". This type of ontology is needed to describe special knowledge about an application or a problem for unit and user. The top-level ontology is oriented for dynamic constraints network, while the lower-level ontology is for ontology-based constraints network.

Knowledge management tools support the conversion of PPR-model, from one ontology to another. An abstract PPR-model is based on the concept of ontologybased dynamic constraint networks. This abstract model unifies main concepts of languages, such as standard object-oriented languages with classes, and constraint programming languages. It supports the declarative representation, efficiency of dynamic constraint solving, as well as problem modeling capability, maintainability, reusability, and extensibility of the object-oriented technology.

The above Ontology Management approach is based on two mechanisms: (1) Object class inheritance mechanism supported by inheritance of class ontologies (attributes inheritance) and by inheritance of constraints on class attribute values, and (2) Constraint inheritance mechanism for inter-ontology conversion supported by constraint inheritance for general model (constraints strengthening for "topdown" or "begin-end" processes).

Multi-agent and Intelligent Agent: The implementation of the basic principle of co-operation in the $\mathrm{SCN}$ is based on distribution of procedures between different units / users (or different agents) concurrently in the common knowledge space. It is, therefore, natural to represent configuration management knowledge as a set of interacting autonomous agents in a multi agent environment. Agent is a software tool that captures behavioral characteristics of the problem for a specific process or activity. Intelligent agent is an autonomous software entity that can navigate heterogeneous computing environment and can, either alone or working with other agents, achieve some goals (Franklin and Graesser, 1996). Integration of agents as cooperative active knowledge processing units organised according to the proposed multilevel Data and Knowledge Management model forms the kernel of the presented approach to SCN configuration management.

Conceptual and Information modeling: In order to design and implement a $\mathrm{SCN}$, it is important to explore and understand its structure and behavior as a system under dynamic environment. Conceptual and information modeling enables representation and evaluation of system entity characteristics, relationships to other entities, and controls to achieve system objectives. Some of the modeling techniques utilized are entity relationships modeling, object-oriented modeling, and computational modeling for evaluation of various enterprise configurations using enterprise-wide database. 
In order to coordinate the flow of material within a multi-level $\mathrm{SCN}$, it is important to synchronize activities within processes both at inter and intra levels by sharing information. To accomplish this objective, it is imperative that activities between trading partners are based on a set of commercial and contractual rules that identify protocols necessary to guarantee cooperation and coordination. To support this objective, an information kernel in the form of a "SCN conceptual model" is needed. This kernel describes the following major components of a SCN:

- A set of objectives at strategic level

- A set of SCN model attributes

- A set of strategies for management at various decision-making levels

- A set of SCN units

- A set of products for every unit
- A set of constraints for every unit

- A set of unit resources

- A set of contract relationships among units

- A set of coefficients for bilateral relationships among units

\section{GENERAL FRAMEWORK OF SCN MANAGEMENT}

The multi-agent approach is much more suited for scalability of networking organization than the conventional approach due to its, (a) orientation towards object-oriented modeling for encapsulation, and (b) suitability for unstructured knowledge problem domains and is advocated as a next generation model for engineering complex, distributed systems (Jennings, 2000). Therefore, a multi-agent approach has been adopted for this paper. An illustration of the information support architecture for a SCN is offered in figure 3.

According to this conceptualization, a supply chain MAS is composed of following two types of agents, each one involving the corresponding unit or group components as depicted in figure 3 :

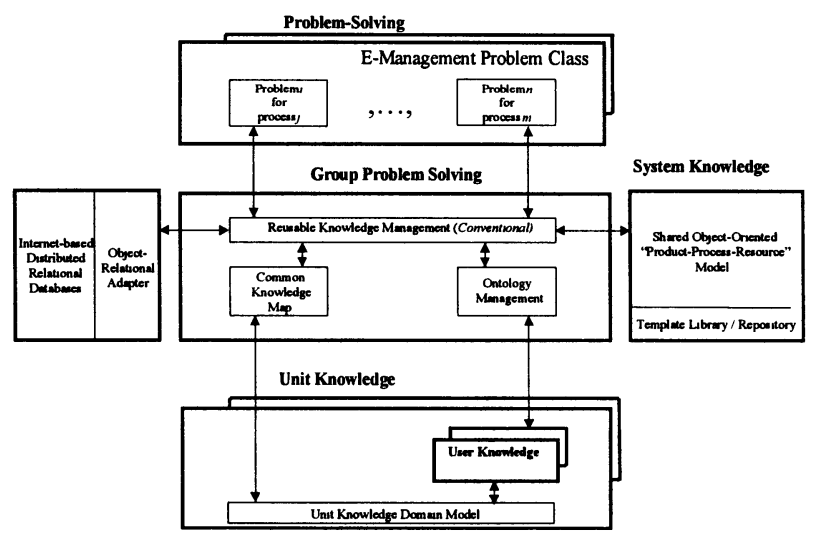

Figure 3. Functional framework for e-management information support of a scalable networking organization

1. Supply chain advisor (SCA) (for each member of the chain). It has following knowledge components: Unit (or Member) knowledge, Resources (Independently owned), Rules (of engagement) for allocation and utilization of resources, Problem Solving methods. 
2. Group supply chain advisor (GSCA). It has following knowledge components: Group's common knowledge, Resources (Commonly shared), Rules (of engagement) for allocation and utilization of (common) resources. GSCA is also oriented for distribution of knowledge and identification of access level in sharing data and knowledge base. It has three major components: (i) conventional reusable knowledge management techniques (Livelink, 1998; and Neches et al., 1991), (ii) common knowledge map that describes distribution of knowledge sources for a SCN, and (iii) ontology management. The last function includes the possibility to convert the PPR from one ontology to another.

An important type of organizational knowledge is about a collection of agents that make up a more complex component, i.e. a compositional relation or "has_a" relation. SCA is the main agent type in the system composed of a number of agents depicted in figure 4. A generic representation of the configuration management problem is based on the interaction of production, inventory and capacity management tasks. Thus, each SCA agent may have its own Problem Solving Agent (PSA) and define its own methods, rules and protocols for problem solving. Each PSA is oriented for a unique class of e-management problem that is applied for problem solving of different management processes described in section 2. For example, in the case of a textile supply chain, a SCA Agent "Retailer" may own its individualized Inventory Management, Production Management, and Capacity Management PSA. These agents are oriented towards application of reusable methods, techniques and solutions for problem solving at the unit level. In the same vein, it is conceivable to have PSAs for forecasting, inventory management and capacity planning for a GSCA. It should be mentioned that at this level, consistency checking, controls of constraints for integration between units of a SCN and its management and maintenance are provided. An example of a PSA, based on constraint satisfaction and propagation techniques is described in (Smirnov and Sheremetov, 1998).

SCA agent also includes the User Interface (UI) and Communication Agent responsible for communication, coordination and negotiation policies. Means of communication are: Protocols (or agreements) for negotiation and compromise between Member Agents and defining each Member Agent's Roles and Responsibilities in Member - to - Group interaction, Methods (trigger policies and strategies) utilized in problem-solving both at the Member Agent and Group Agent levels, and Services. For this agent following negotiation algorithms are implemented: CNP, Auctions, Multistage negotiation algorithm (Sheremetov and Núñez, 1999).

These two types of agents form the SCN agent domain. The framework of normative work, inside which the agents exist and operate, as well as the logical and temporal contexts for the creation, operation and destruction of agents are provided by the Agent Platform (AP) (FIPA, 1998). The agent platform developed in this project to implement a multi-agent platform based on the DCOM model is depicted in figure 5 and consists of following types of agents:

- Management agent. The AMS is responsible for managing activities of agents of an AP. For this purpose, it maintains a permanent list of all resident agents. 
This list includes at least the agent's unique name and its address. Each AP must have only one AMS.

- Domain facilitator (DF) agent. While GSCA provides the system with domain shared knowledge (rules, resources and constraints), DF is responsible for service registration (roles) of SCA agents (yellow pages), it also facilitates communication between them (in the phase of a peer-to-peer connection establishment). The DF maintains a list of services that each agent provides, and additional characteristics as agent's type, its state and owner. For each service, among other things, their name, their type, and their ontology are stored. The agents communicate with the DF either to register their own services or to find out about services that other agents have to offer. A group of agents registered in a DF is known as agent domain. There should exist at least one DF in each SCN.

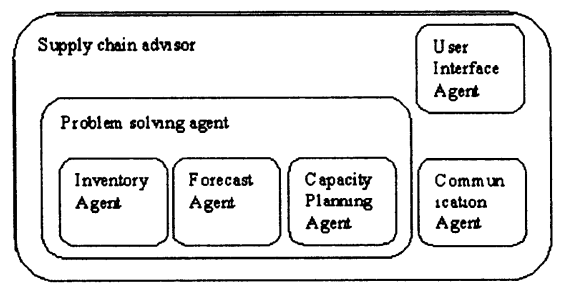

Figure 4. Supply chain advisor structure

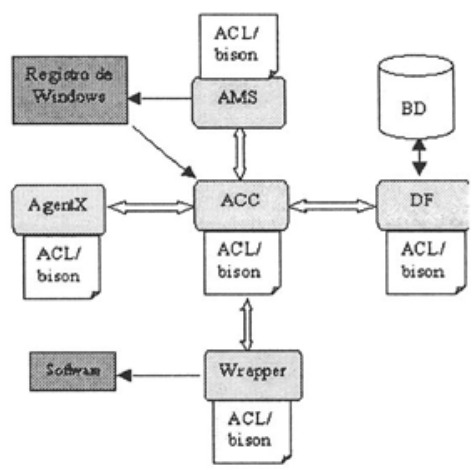

(Thick lines indicate communication among agents, while thin lines depict flow of information.)

Figure 5. Architecture of the Agent Platform.

- Communication channel. The ACC is a way by which an agent is able to communicate with other agents, including the AMS and the DF. So, each agent should have access to at least one ACC. The ACC not only routes messages inside a platform, but is also capable of routing messages to an ACC in another platform.

- Wrapper agent (W). This agent facilitates access to the MAS and enterprise databases.

The communication language between agents of the AP is a subset of the Agent Communication Language (ACL) proposed by FIPA. On the other hand, since the grammar of the language may be rather complicated, each agent makes use of a parser-generating tool, called bison. 


\section{MULTIAGENT SYSTEM DESIGN AND IMPLEMENTATION}

The deployment of agents of the SCN MAS occurs in the realm of a problemsolving environment according to the architecture depicted earlier in figure 5. Each agent is characterized in terms of services and actions. In the case of a SCA, tables 1 and 2 describe list of services and actions that can be requested in the SCA Forecast and Inventory ontologies. A description of each action including the agent that supports the action; the content of the action, i.e. its parameters, and the interaction protocol are provided below through an example.

Table 1-Service Description Attributes

\begin{tabular}{|c|c|c|}
\hline Parameter & Description & Pre-defined constants \\
\hline $\begin{array}{l}\text { :service-name } \\
\text { generateDemandData } \\
\text { generateForecast } \\
\text { selectAlgorithm }\end{array}$ & $\begin{array}{l}\text { Denotes the service name. } \\
\text { Fetch historic and current demand data, } \\
\text { and prepare demand time series. } \\
\text { Utilizing demand data generate a } \\
\text { forecast time series. } \\
\text { Based on demand pattern depicted by } \\
\text { time series, choose appropriate forecast } \\
\text { algorithm. }\end{array}$ & $\begin{array}{l}\text { Following data are used for } \\
\text { service description: } \\
\text { Planning horizon: time } \\
\text { (period), } t=1 \text { to } 52 \\
\text { (Sales) Demand by period: } d_{t} \\
\text { Forecast by period: } f_{t} \\
\text { Replenishment by period: } r_{t} \\
\text { Forecast error }\left(\varepsilon_{t}\right): f_{t}-d_{t}\end{array}$ \\
\hline $\begin{array}{l}\text { :service-type } \\
\text { Data retrieval } \\
\text { Data manipulation } \\
\end{array}$ & Identifies type of service described: & \\
\hline $\begin{array}{l}\text { :service-ontoloğy } \\
\text { ForeacastManagement } \\
\text { InventoryManagement }\end{array}$ & $\begin{array}{l}\text { Denotes the ontology(ies) the service } \\
\text { can support. }\end{array}$ & \\
\hline $\begin{array}{l}\text { :fixed-properties } \\
\text { forecast_error_max }\end{array}$ & $\begin{array}{l}\text { Denotes a list of fixed, i.e. static and } \\
\text { non-negotiable properties of the service }\end{array}$ & \\
\hline $\begin{array}{l}\text { :negotiable-properties } \\
\text { forecast_error_min cost } \\
\text { price }\end{array}$ & $\begin{array}{l}\text { Denotes a list of properties whose value } \\
\text { can be determined dynamically or the } \\
\text { broker agent may wish to negotiate. }\end{array}$ & \\
\hline $\begin{array}{l}\text { :communication-properties } \\
\text { FIPA-Iterated-CNP } \\
\text { FIPA-Auction } \\
\text { Multistage Algorithm }\end{array}$ & $\begin{array}{l}\text { Identifies the unique address of the } \\
\text { software system described by this } \\
\text { software description, as well as the } \\
\text { networking protocol to be used when } \\
\text { interfacing with the software system. }\end{array}$ & \\
\hline
\end{tabular}

Table 2-Actions

\begin{tabular}{|l|l|}
\hline Action & Agent \\
\hline InitiateDemandForecast & ProblemSolvingAgent: sub-agent Forecast Management \\
\hline GenerateDemandData & Forecast Agent: sub-agent Demand Data \\
\hline GenerateForecast & Forecast Agent: sub-agent Forecast Demand \\
\hline SelectAlgorithm & Forecast Agent: sub-agent Forecast Demand \\
\hline UpdateForecast & Forecast Agent: sub-agent Forecast Demand \\
\hline BroadcastDemandData & Communication Agent \\
\hline
\end{tabular}


Services of agents' management that the AP offers are grouped according to the component that provide the services. For more details see (Sheremetov and González, 2000). All offered services are invoked by sending a message in language SL0 to the corresponding agent. The implementation of the communication language permits defining multiple ontology in the message parameter section.

All agents of this architecture have been created using Visual $\mathrm{C}++$. To build SCN agents, a template project of a Client Agent was created. Supply chain agents sub-classing is depicted in figure 6. For those agents that need to receive messages for their operation, a file in bison format that exemplifies the interpretation of ACL messages is included in the project of the AMS.

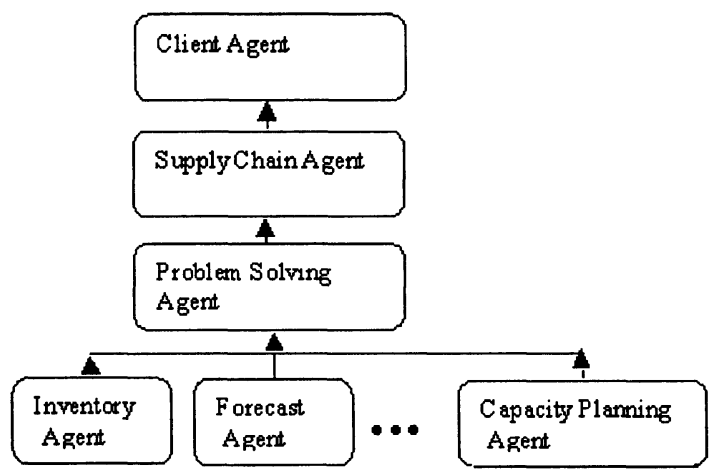

Figure 6. Supply chain agent sub-classification

\section{MULTIAGENT SYSTEM INTERACTION SCENARIOS}

Interactions between agents are considered as one of the key issues of the MAS software engineering (Jennings, 2000). SCA interactions to synchronize demand and supply echelons are depicted in figure 7. GSCA and DF act as mediators between these Member agents. Mediation occurs when services are requested by one Member from another.

In order to solve a problem, the PSA through the DF deploys reusable knowledge management to communicate with shared object-oriented data model (based on template libraries and repositories) and ontology-based / user-oriented knowledge domain models of SCA. Unit knowledge domain model describes the environment at the unit level, such as objectives, constraints and resources. GSCA utilizes the shared object-oriented data model for consistency checks of unit's data and knowledge for problem solving. It communicates through the wrapper agent with Internet-based distributed relational databases that describe the dynamic environment of a SCN.

A real demand such as a point-of-sale data from the $S C A_{a}$ agent, causes the echelons re-configuration and a new set of forecasts is generated. MAS evaluates demand and broadcasts these across the supply chain; reconfigures limits of negotiation for members to commit to this new set of variables. Three agents, $S C A_{b}$, 
$S C A_{c}$ and $S C A_{d}$ participate in the negotiation in the following scenario, which is

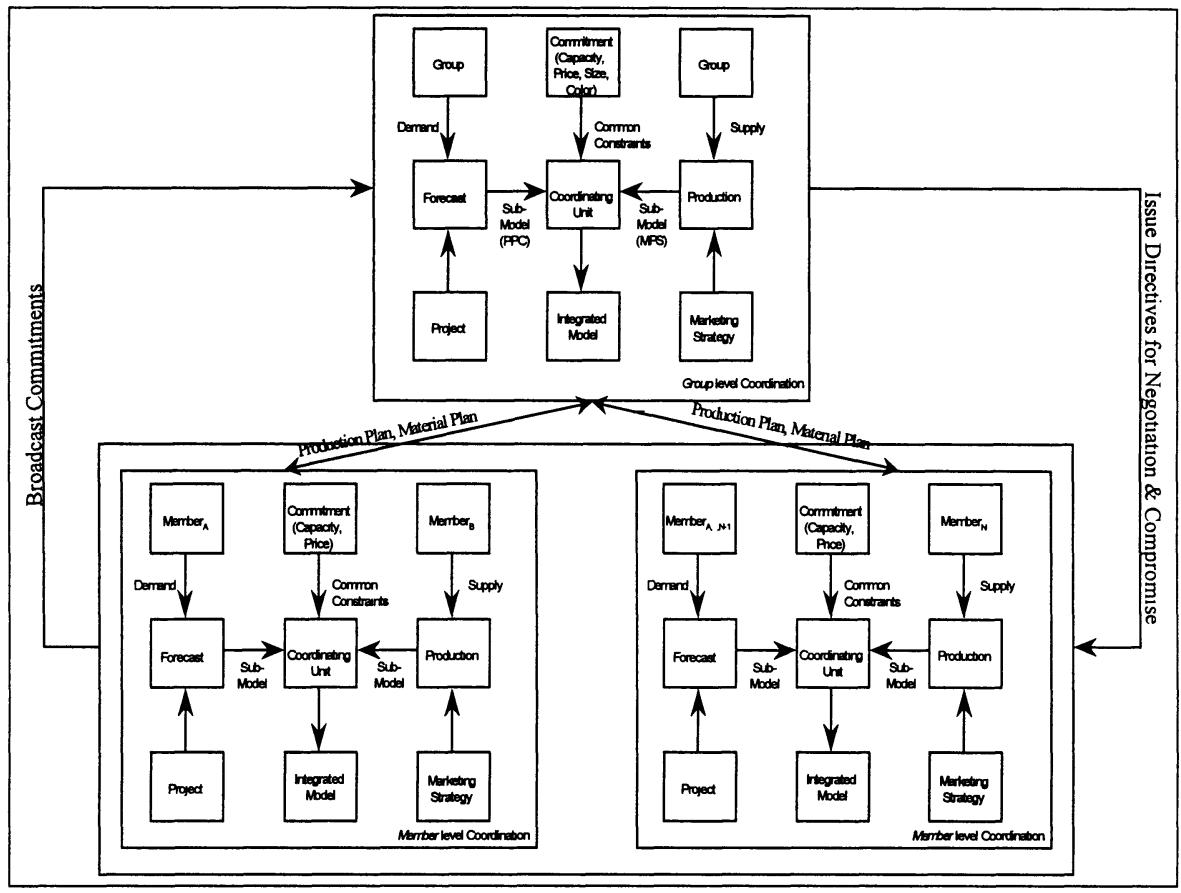

Figure 7. Member SCA agent - to - GSCA agent interaction to synchronize demand and supply echelons across the supply chain

partially supported by the agent interaction diagram and FIPA iterated CNP described below:

$S C A_{a} \rightarrow G S C A$

Inform (time- series- data): Communication of time series data

$G S C A \rightarrow S C A_{b} d$

$c f p$ (demand forecast, time- series- data): Communication of time series demand data to all agents in the supply chain

$S C A_{b} \rightarrow G S C A$

propose (time series replenishment data): Express intentions to buy goods / and or services $S C A_{c} \rightarrow G S C A$

propose (time series replenishment data): Express intentions to buy goods / and or services $S C A_{d} \rightarrow G S C A$

refuse (demand forecast, conflict(planned capacity, time- series- data)): Express a conflict; planned capacity can't meet Agent A's demand, Agent D will not participate in the new SCN configuration. 
$G S C A \rightarrow S C A_{b}$

accept-proposal (planned capacity): signify final commitment, planned capacity to meet Agent A's demand

$G S C A \rightarrow S C A_{c}$

reject-proposal (conflict(time series replenishment data, time- series- data)): signify a conflict; proposal can't meet Agent A's demand

$S C A_{c} \rightarrow G S C A$

propose (new time series replenishment data): Agent $\mathrm{C}$ changes its conditions

$G S C A \rightarrow S C A_{c}$

accept-proposal (planned capacity): signify final commitment, new conditions are accepted, planned capacity to meet Agent A's demand

$S C A_{b} \rightarrow G S C A$

Inform (Done(time series replenishment data)): signify commitments to meet replenishment schedule, planned replenishments to meet Agent A's demand

$S C A_{c} \rightarrow G S C A$

Inform (Done(time series replenishment data)): signify commitments to meet replenishment schedule, planned replenishments to meet Agent A's demand. Final capacity agreements in the supply chain network

\section{CONCLUSIONS AND EXTENSIONS}

A model, architecture and a multi-agent platform for SCN configuration management has been developed and described in this paper. The MAS architecture is recommended where SCA agents form a dynamic network of flexible problem solvers, able to dynamically create the organizational structure, and the flexible interaction structure guaranteed by the AP. In the current phase of experimentation, work on the AP has been completed, while problem solving agents of the SCA that use the platform presented in this paper are being tested.

This platform is an open system of complex software based on the FIPA reference model and DCOM. All components were programmed as executables, because each one of them forms an application.

Current experiments are focused on the development of the SCA and testing, as well as on further investigation of the interoperability issues with software modules that provide additional services for agents. Among scenarios that are being experimented to observe behavior of the system -- each member advisor agent looks for the authorization of the corresponding user while negotiating the contract terms, or the system has a unique user responsible for the SC topology construction, while limits of parameters to be negotiated are defined within advisor agents. 


\section{ACKNOWLEDGEMENTS}

Components of the above approach have been developed for the "Demand Activated Manufacturing Architecture project for the United States Integrated Textile Complex", supported by grant \# H1757-0019-2G 11/9/98, to the first author from Los Alamos National Laboratory, United States Department of Energy. The project, "Affordable Cost Structure", from Ford Motor Company supported work of the second author. Partial support for this work for the third author was also obtained from CONACyT, Mexico within the project 31851-A "Models and Tools for Agent Interaction in Cooperative MAS".

\section{REFERENCES}

Banerjee SK, Smirnov A. Manufacturing Management and Manufacturing Strategy: an Agent-based Support System. Proceedings of the International Conference on Industrial Engineering and Production Management (IEPM'99), Glasgow, July 12-15, 1999, 453-463.

Chandra, C, Kumar, S. Enterprise Architectural Framework for Supply-Chain Integration, Journal of Industrial Management and Data Systems, 2000 (forthcoming).

Chen, F. Decentralized supply chains subject to information delays, Management Science, 1999, 45(8): 1076-1090.

(FIPA, 1998) FIPA '97 Draft Specification version 2.0: Agent Management, Foundation for Intelligent Physical Agents, URL: http://www.fipa.org.

Franklin, S, Graesser, A. Is it an agent or just a program?: A taxonomy for autonomous agents. Proceedings of the Third International Workshop on Agent Theories, Architectures, and Languages, Springer-Verlag, 1996.

Groumpos P, Smirnov. A. Multi-Agent Based Virtual Enterprise Configuration Management: General Principles and Basic Technologies. Large Scale Systems: Theory \& Applications: Proceedings of the 8th IFAC/IFORS/IMACS/IFIP Symposium LSS'98, Patras, Greece, 1998, 547-552.

(IMTR, 1999) Technologies for Enterprise Integration, Rev 3.1 (Oct 99), Integrated Manufacturing Technology Roadmapping Project, Oak Ridge Centers for Manufacturing Technology, Oak Ridge, Tennessee. URL: http://imti21.org.

ISO TC 184/SC 5/WG 1 (1997) Requirements for enterprise reference architectures and methodologies, http://www.mel.nist.gov/sc5wgl/gera-std/ger-anxs.html]

Jennings, NR. On agent-based software engineering. Artificial Intelligence, 2000, 117:277-296.

Livelink: Collaborative Knowledge Management, http://www.opentext.com/livelink/ knowledge_management.html, 1998.

Moulin, B, Chaib-draa, B. An Overview of Distributed Artificial Intelligence. In Greg O'Hare \& Nick Jennings (Eds.) Foundations of Distributed Artificial Intelligence, John Wiley \& Sons, 1996.

Neches, R, Fikes, RE, Finin, T, Gruber, T, Patil, R, Senator, T, Swartout, WR. Enabling Technology for Knowledge Sharing, AI Magazine, 1991, 12(3):16 - 36.

(NIST 1999) Manufacturing Enterprise Integration Program (Nov 99), National Institute of Standards and Technology, Gaithersburg, Maryland. (URL: http://www.atp.nist.gov)

Sheremetov, L. Núnez, G. Multi-Stage Cooperation Algorithm and Tools for Agent-Based Planning and Scheduling in Virtual Learning Environment, The First International Workshop of Central and Eastern Europe on Multi-agent Systems (CEEMAS'99), 30th May-3rd June 1999, St. Petersburg, Russia, 211-223.

Smirnov, AV, Sheremetov, LB. Configuration of complex systems based on the technology of intelligent agents. Automatic Control and Computer Sciences, Allerton Press, N.Y., 1998, 32(4):15-24.

Sousa, P, Heikkila, T, Kollingbaum, M, Valckenaers, P. Aspects of co-operation in Distributed Manufacturing Systems, In: Proceedings of the Second International Workshop on Intelligent Manufacturing Systems, September 1999, Leuven, Belgium, 685-717

Sheremetov, L, González, J. Desarrollo y Construcción de una Plataforma Multiagente Basada en el Modelo de Referencia de FIPA y DCOM., Simposium Internacional de Sistemas Distribuidos Avanzados (SISDA), CD ROM, 2000, (in Spanish). 